NEUROLOGICAL PICTURE

\title{
Eye movement abnormality associated with cyclosporin
}

A 50 year old man developed diplopia 2 months after an allogeneic bone marrow transplantation for mantle cell lymphoma. He was receiving cyclosporin for graft versus host disease prophylaxis and ganciclovir for a positive cytomegalovirus blood culture. Neurological examination within 24 hours of developing diplopia showed apathy, distractibility, and a postural tremor. He tended to keep his eyes closed. Visual acuity was 20/400 in both eyes. There was bilateral weakness in eye abduction with the left eye not crossing, and the right eye barely crossing the midline. Brain MRI showed patchy high signal abnormality on the T2 weighted and (as shown) FLAIR sequences, particularly in the subcortical white matter of the occipital lobes but also in the brain stem and the cerebellar vermis. Cyclosporin was stopped and within 2 days eye movements returned to normal. A repeat MRI 2.5 weeks later showed almost complete resolution of the high signal abnormality.

Cyclosporin is a common cause of seizures and encephalopathy in transplant patients. ${ }^{1}$ Occasionally with cyclosporin neurotoxicity, white matter abnormalities occur producing transient cortical blindness as the most usual clinical manifestation. ${ }^{2}$ Four patients were reported in whom cyclosporin caused transient unilateral or bilateral sixth nerve palsies. ${ }^{3}$ All four of these patients (as the patient shown here) were also on ganciclovir, and one of these patients had white matter changes in the cerebellum associated with cyclosporin. The MRI presented here, clearly showing abnormalities in the region of the sixth nerve nucleus (arrow head), argues in favour of a CNS rather than a neuromuscular localisation of the eye movement abnormality. These patients show that development of a sixth nerve palsy in a patient with a bone marrow transplant does not necessarily mean a leptomeningeal recurrence of the haematological malignancy.

H OPENSHAW

City of Hope National Medical Center, Department of Neurology, 1500 East Duarte Road, Duarte, California 91010, USA

hopenshaw@coh.org

1 Reece DE, Frei-Lahr DA, Shepherd JD, et al. Neurologic complications in allogeneic bone marrow transplant patients receiving cyclosporin. Bone Marrow Transplant 1991;8:393-401.

2 Truwit CL, Denaro CP, Lake JR, et al. MR imaging of reversible cyclosporin A-induced neurotoxicity. Am $\mathcal{F}$ Neuroradiol 1991;12:651-9.

3 Openshaw H, Slatkin NE, Smith E. Eye movement disorders in bone marrow transplant patients on cyclosporin and ganciclovir. Bone Marrow Transplant 1997;19:503-5.

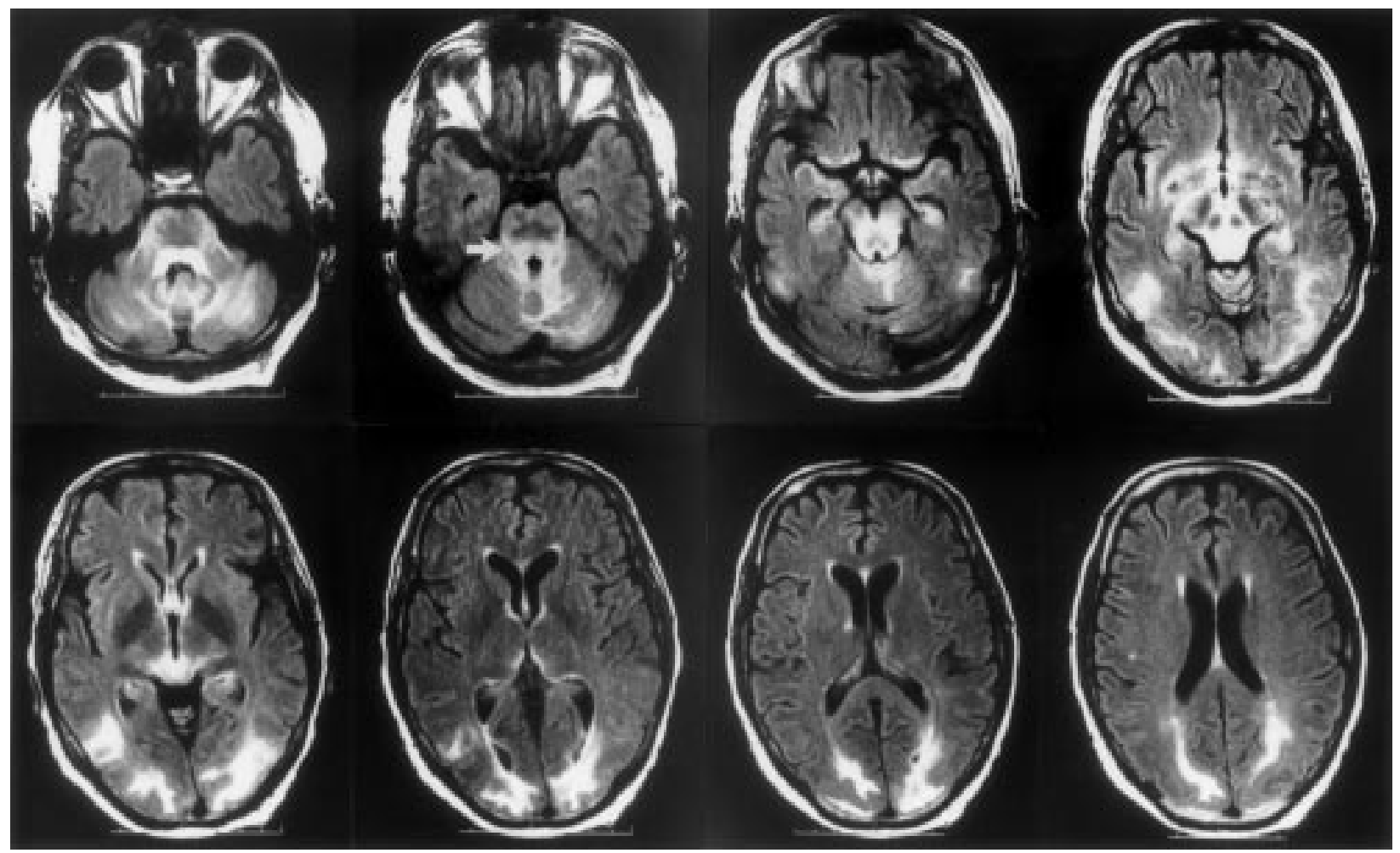

